\title{
Kanglaite inhibits the expression of drug resistance genes through suppressing PVT1 in cisplatin-resistant gastric cancer cells
}

\author{
XIAN-WEN ZHANG ${ }^{1,2^{*}}$, LIANG LIU $^{1 *}$, XI-ZHI ZHANG ${ }^{2}$ and PING BO ${ }^{1}$ \\ ${ }^{1}$ Medical College, Yangzhou University; ${ }^{2}$ Department of Oncology, Northern \\ Jiangsu People's Hospital, Yangzhou, Jiangsu 225009, P.R. China
}

Received December 7, 2016; Accepted June 1, 2017

DOI: $10.3892 /$ etm.2017.4650

\begin{abstract}
Kanglaite (KLT) was shown to alleviate the development of multidrug resistance (MDR) clinically. The purpose of this study is to examine the mechanism of KLT for chemotherapy resistance in gastric cancer cells involving the regulation of MDR-related proteins. The cisplatin-resistant BGC823/DPP and SGC7901/DDP cells were treated with 1, 2.5 and $5 \mu \mathrm{l} / \mathrm{ml}$ of KLT for 24, 36 and $48 \mathrm{~h}$. Cell Counting Kit-8 (CCK-8) assay and flow cytometry were performed to detect the cell viability and cell apoptosis, respectively. The expression of MDR1 and multidrug resistance associated protein 1 (MRP1) were examined by quantitative PCR and western blotting in BGC823/DPP cells and SGC7901/DDP cells treated with KLT. The effect of KLT on the expression of PVT1 was investigated. PVT1-overexpression vector was constructed and transfected into BGC823/DPP cells and SGC7901/DDP cells which were treated with KLT. KLT inhibited the cell viability and promoted the cell apoptosis of BGC823/DPP cells and SGC7901/DDP cells in a concentration-dependent manner. KLT suppressed the expression of MDR1 and MRP1 and the level of PVT1. PVT1 overexpression reversed the increased percentage of apoptotic cells induced by KLT. Finally, we found that PVT1 overexpression also abrogated the effect of KLT on the mRNA level and protein level of MDR1 and MRP1 in BGC823/DPP and SGC7901/DDP cells. KLT inhibited the expression of MDR1 and MRP1 via suppressing the expression of PVT1, which suggested the potential mechanism of KLT involving in MDR in gastric cancer.
\end{abstract}

Correspondence to: Dr Ping Bo, Medical College, Yangzhou University, 11 Huaihai Road, Yangzhou, Jiangsu 225009, P.R. China E-mail: pingB2250@163.com

*Contributed equally

Key words: Kanglaite, drug resistance, PVT1, gastric cancer, MDR1, MRP1

\section{Introduction}

Gastric cancer is one of the most common malignant carcinoma in the world. Currently, the approaches for gastric cancer therapy mainly included surgical therapies, radiotherapies and chemotherapies. However, the development of multidrug resistance (MDR) greatly decreased the efficacy of chemotherapies. Several studies suggested that attenuating the development of MDR could restore the sensibility of cancer cells to chemotherapies $(1,2)$. Specially, the expression of MDR-related proteins (MDR1, MRP1) played crucial roles in the development of MDR (3). MDR1 and MRP1 belong to the ATP-binding cassette (ABC) superfamily, which mediated the efflux of drugs out of cells, reducing drug efficacy.

Kanglaite (KLT) is one of Chinese herb injection reported to show antitumor activity (4). KLT is a coix seed oily substance extracted from Coix lacryma-jobi (family Cramineae). The main active ingredient of coix seed is a compound of triglycerides containing four types of fatty acid (5). The coix seed oil is well known for its antitumor and immunomodulatory effects among traditional Chinese medicine (6). In 1997, KLT injection was officially approved by the Ministry of Public Health in China for the treatment of liver cancer, lung cancer and gastric cancer $(6,7)$. Moreover, KLT has showed good efficacy in the USA and its clinical trial smoothly completed in Russia, where it has been registered as a new drug and can be marketed legally (8). In later research, KLT was found to ameliorate MDR of cancers when combined with radiotherapy and chemotherapy in clinical use (9). For example, Zhan et al reported that patients with gastric cancer treated with KLT injection combined with chemotherapy showed lower gastrointestinal reactions and bone marrow suppression than that in the patients with chemotherapy alone, which indicated that KLT injection enhanced efficacy and reduced the side effects of chemotherapy (10). However, the mechanism of KLT working against chemotherapy resistance in gastric cancer cells involving the regulation of MDR-related proteins was poorly understood.

PVT1 is a long non-coding RNA located in human chromosome 8q24 (11). To date, overexpression of PVT1 has been frequently observed in several malignant diseases, such as breast cancer, colorectal cancer, ovarian cancer and gastric cancer, and is associated with increasing cell proliferation and inhibiting cell apoptosis (12-14). Recently, mounting 
evidence indicated that PVT1 participated in the drug resistance of cancer cells by regulating different pathways $(15,16)$. In our previous study, we also found that overexpression of PVT1 promoted the development of MDR in gastric cancer cells (16). Based on these data, in the present study, we further investigated the role of PVT1 in the effect of KLT on drug resistance in gastric cancer cells, which might shed light on elucidating the potential mechanism of KLT in ameliorating MDR of cancer cells.

\section{Materials and methods}

Cell lines and culture. The cisplatin-resistant BGC823/DDP cells and SGC7901/DDP cells were obtained as the previous study (16). Briefly, human gastric cancer cell lines BGC823 and SGC7901 obtaining from the Type Culture Collection of the Chinese Academy of Sciences (Shanghai, China) were exposed to cisplatin with gradually increasing concentration for about 12 months. The cisplatin concentration was from $0.05 \mathrm{mg} / \mathrm{ml}$ until the cells acquired resistance to $1 \mathrm{mg} / \mathrm{ml}$. Finally, the cisplatin-resistant BGC823/DDP cells and SGC7901/DDP cells were developed. Cells were cultured in RPMI-1640 medium (Gibco; Thermo Fisher Scientific, Inc., Waltham, MA, USA) supplemented with $10 \%$ of fetal bovine serum (Gibco; Thermo Fisher Scientific, Inc.), $100 \mathrm{U} / \mathrm{ml}$ of penicillin and $100 \mathrm{mg} / \mathrm{ml}$ of streptomycin (Gibco; Thermo Fisher Scientific, Inc.) at $37^{\circ} \mathrm{C}$ in a humidified incubator with $5 \% \mathrm{CO}_{2}$.

Plasmids and cell transfection. PVT1-overexpression vector (Ad-PVT1) was constructed and synthetized by Ribobio Co., Ltd. (Guangzhou, China). BGC823/DDP and SGC7901/DDP cells were transfected Ad-PVT1 by using Lipofectamine 2000 transfection reagent (Invitrogen Life Technologies, Carlsbad, CA, USA) according to the manufacturer's instructions.

Cell Counting Kit-8 (CCK-8) assay. CCK-8 assay was performed to detect the effect of KLT on the cell viability of BGC823/DDP cells and SGC7901/DDP cells. KLT ${ }^{\circledR}$ (Coix Seed Oil) injection $(10 \mathrm{~g} / 100 \mathrm{ml})$ and blank emulsion (as vehicle) were obtained from the Zhejiang Kanglaite Pharmaceutical Co., Ltd. (Hangzhou, China). The cells $\left(5 \times 10^{3}\right.$ cells $\left./ \mathrm{ml}\right)$ were cultured on a 96-well plate in a RPMI-1640 medium supplemented with different concentrations of KLT $(1,2.5$ and $5 \mu \mathrm{l} / \mathrm{ml}$ ) for 24,36 and $48 \mathrm{~h}$. After the incubation, CCK- 8 was added into each well, followed by incubation for $1 \mathrm{~h}$ in humid atmosphere containing $5 \% \mathrm{CO}_{2}$. Absorbance was determined at $450 \mathrm{~nm}$ by microplate reader.

Apoptosis analysis by flow cytometry. An Annexin V-FITC Apoptosis Detection kit I (BD Pharmingen, San Diego, CA, USA) was used to examine the cell apoptosis according to the manufacturer's instructions. Cells were cultured on a 96-well plate in a RPMI-1640 medium supplemented with different concentrations of $\operatorname{KLT}(1,2.5,5 \mu \mathrm{l} / \mathrm{ml})$ for $48 \mathrm{~h}$. Then cells were washed with PBS and resuspended in $1 \mathrm{X}$ binding buffer at a concentration of $1 \times 10^{6}$ cells $/ \mathrm{ml}$. Cell suspension $(100 \mu \mathrm{l})$ was incubated with $5 \mathrm{ml}$ of FITC Annexin-V and $5 \mu \mathrm{l}$ of propidium iodide (PI) for $15 \mathrm{~min}$ in the dark. Following the incubation, $400 \mu 11 \mathrm{X}$ binding buffer was added. Flow cytometry was used to analyze the cell apoptosis with the EPICS XL-MCL FACScan (BD Biosciences, Mountain View, CA, USA). Annexin V-FITC-positive and PI-negative cells indicated the cells undergoing apoptosis.

Western blot analysis. RIPA buffer was used to extract the total protein from cell lysates and the protein concentration was measured by BCA Protein Assay Kit (Pierce Chemical Co., Ltd., Rockford, IL, USA). Then proteins $(20 \mu \mathrm{g})$ were separated by $12 \%$ SDS-polyacrylamide gel electrophoresis and transferred onto PVDF membranes (Millipore Corp., Bedford, MA, USA). After blocking with $10 \%$ dry milk in TBST for $1 \mathrm{~h}$ at room temperature, membranes were incubated with antibodies against MDR1 $(1: 3,000)$ and MRP1 $(1: 1,500)$, or anti-GAPDH $(1: 5,000)$ as a control overnight at $4^{\circ} \mathrm{C}$. Blots were incubated with a peroxidase-linked secondary antibody $(1: 1,000)$ for $1 \mathrm{~h}$. The enhanced chemiluminescence (ECL) detection system (Amersham Biosciences, Uppsala, Sweden) was used to detect the antibody binding.

Quantitative PCR. Quantitative PCR was performed to detect the level of PVT1 and the mRNA level of MDR1 and MRP1. Briefly, total RNA from cultured cells was extracted by using TRIzol reagent (Invitrogen Life Technologies) according to the manufacturer's instructions. Then the total RNA was used as the templet to convert into cDNA using the RevertAidHMinus First Strand cDNA synthesis kit (Fermentas; Thermo Fisher Scientific, Inc., Waltham, MA, USA). A SYBR-Green PCR Mastermix was used to perform the quantitative PCR on an Mx3000P Real-Time PCR system (Stratagene; Agilent Technologies, Inc., Santa Clara, CA, USA). PCRs were performed with the following primer sets: PVT1 forward, 5'-CAGCACTCTGGACGGAC-3' and reverse primers, 5'-CAACAGGAGAAGCAAACA-3'; MDR1 forward, 5'-ACC AAGCGGCTCCGATACA-3' and reverse primers, 5'-TCA TTGGCGAGCCTGGTAGTC-3'; MRP1 forward, 5'-GGA CCTGGACTTCGTTCTCA-3' and reverse primers, 5'-CGT CCAGACTTCATCCG-3'; GAPDH forward, 5'-ACCACA GTCCATGCCATCAC-3' and reverse primers, 5'-TCACCA CCCTGTTGCTGTA-3'. RNA levels were corrected with the GAPDH signal. All mRNA levels were calculated using the $2^{-\triangle \Delta C q}$ method.

Statistical analysis. The values were expressed as the mean \pm standard deviation (SD). SPSS 17.0 software was used to analyze the data. The Student's t-test was performed to detect differences between the KLT and vehicle groups. $\mathrm{P}<0.05$ was considered significant.

\section{Results}

KLT inhibited the cell viability and promoted the cell apoptosis of drug-resistant gastric cancer cells. BGC823/DPP cells and SGC7901/DDP cells were treated with $1,2.5$ and $5 \mu \mathrm{l} / \mathrm{ml}$ of KLT for 24,36 and $48 \mathrm{~h}$. The results of CCK8 assay showed that the cell viability of BGC823/DPP cells and SGC7901/DDP cells was significantly decreased by KLT in a concentration-dependent manner (Fig. 1A and B). To detect the effect of KLT on cell apoptosis of drug-resistant gastric cancer cells, BGC823/DPP cells and SGC7901/DDP 
A

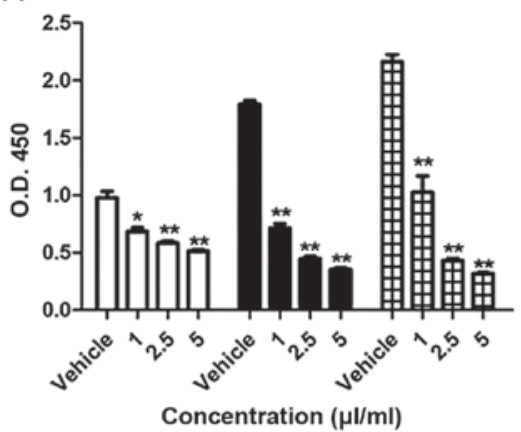

B

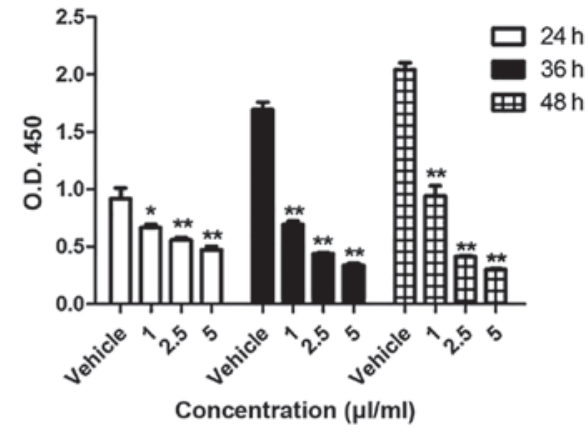

C

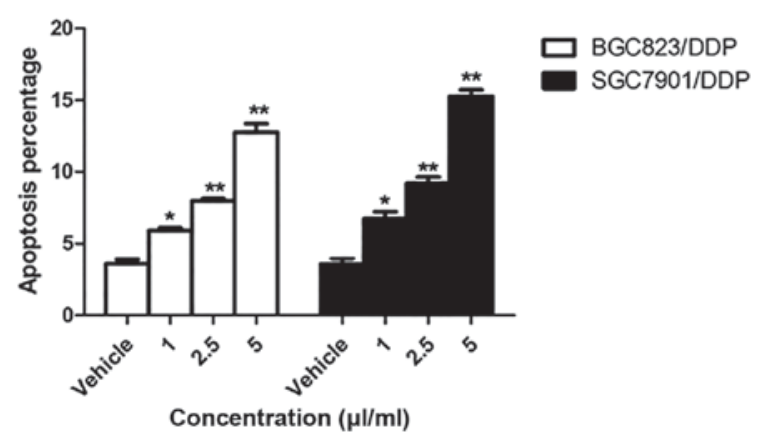

Figure 1. KLT inhibited the cell viability and promoted the cell apoptosis of drug-resistant gastric cancer cells. Different concentration (1, 2.5 and $5 \mu 1 / \mathrm{ml})$ of KLT were used to treat BGC823/DPP cells and SGC7901/DDP cells for 24,36 and $48 \mathrm{~h}$. CCK8 assay was performed to detect the cell viability of BGC823/DPP cells (A) and SGC7901/DDP cells (B). BGC823/DPP cells and SGC7901/DDP cells were treated with different concentration (1, 2.5 and 5 $\mu 1 / \mathrm{ml})$ of KLT for $48 \mathrm{~h}$, and the cell apoptosis was detected by Annexin V-PI assay $(\mathrm{C})$. All values are mean $\pm \mathrm{SD}$. " $\mathrm{P}<0.05$ vs. vehicle group; ${ }^{* *} \mathrm{P}<0.01$. vs. vehicle group.

cells were treated with $1,2.5$ and $5 \mu \mathrm{l} / \mathrm{ml}$ of KLT for $48 \mathrm{~h}$ and detected by Annexin V-PI assay. As shown in Fig. 1C, KLT highly promoted the cell apoptosis of BGC823/DPP cells and SGC7901/DDP cells which was also dose-dependent. Particularly, BGC823/DPP cells or SGC7901/DDP cells treated with $5 \mu \mathrm{l} / \mathrm{ml}$ of KLT showed 3.5 -fold increase or 4.2-fold increase of apoptotic percentage compared to cells treated with vehicle. These results indicated that KLT inhibited the cell viability and promoted the cell apoptosis of drug-resistant gastric cancer cells.

KLT suppressed the expression of drug resistance-related proteins. As drug resistance-related proteins might contribute to MDR, we detected the mRNA levels of MDR1 and MRP1 by means of Real Time-PCR in BGC823/DPP and SGC7901/DDP cells treated with different concentration (1, 2.5, $5 \mu \mathrm{l} / \mathrm{ml}$ ) of KLT for $24 \mathrm{~h}$. As shown in Fig. 2A and B, the expression of MDR1 and MRP1 were both reduced by the pretreatment of KLT in a concentration-dependent manner in BGC823/DPP and SGC7901/DDP cells. Compared to the control (vehicle) the expression of MDR1 and MRP1 was 51 and $49 \%$ in BGC823/DPP cells treated with $5 \mu \mathrm{l} / \mathrm{ml}$ of KLT, respectively. Then western blot was performed to examine the protein level of MDR1 and MRP1 in BGC823/DPP and SGC7901/DDP cells treated with $2.5 \mu \mathrm{l} / \mathrm{ml}$ of KLT for $48 \mathrm{~h}$. KLT also greatly decreased the protein level of MDR1 and MRP1 (Fig. 2C). These data indicated that KLT suppressed the expression of drug resistance-related proteins.

KLT inhibited the expression of PVT1 in drug-resistant gastric cancer cells. To investigate the effect of KLT on the expression of PVT1 in gastric cancer cells, the level of PVT1 was detected in BGC823/DPP and SGC7901/DDP cells treated with $1,2.5$ and $5 \mu \mathrm{l} / \mathrm{ml}$ of KLT for $24 \mathrm{~h}$. As shown in Fig. 3A, significant downregulation of PVT1 was observed in all Kanglaite-treated groups vs. the vehicle groups. Then BGC823/DPP and SGC7901/DDP cells were transfected Ad-PVT1 followed by the treatment of $2.5 \mu \mathrm{l} / \mathrm{ml}$ of KLT for $48 \mathrm{~h}$. The result of cell apoptosis showed that compared to BGC823/DPP and SGC7901/DDP cells treated with KLT alone, the apoptotic percentage was 46 and 50\% in cells treated with KLT and Ad-PVT1, which indicated that PVT1 overexpression reversed the increased percentage of apoptotic cells induced by KLT (Fig. 3B).

KLT inhibited the expression of drug resistance-related proteins via suppressing the expression of PVT1. BGC823/DPP and SGC7901/DDP cells were transfected Ad-PVT1 and then treated with $2.5 \mu \mathrm{l} / \mathrm{ml}$ of KLT for $24 \mathrm{~h}$. The mRNA level of MDR1 and MRP1 were detected by Real Time-PCR and the results showed that PVT1 overexpression increased the mRNA level of MDR1 and MRP1 which was inhibited by KLT (Fig. 4A and B). For example, BGC823/DPP cells treated with KLT and Ad-PVT1 showed 1.66 -fold increase of MDR1 expression and 1.57-fold increase of MRP1 expression compared to cells treated with KLT alone. In addition, PVT1 overexpression also abrogated the effect of KLT on the protein level of MDR1 and MRP1 in BGC823/DPP and SGC7901/DDP cells (Fig. 4C). These data suggested that KLT inhibited the expression of drug resistance-related proteins via suppressing the expression of PVT1. 

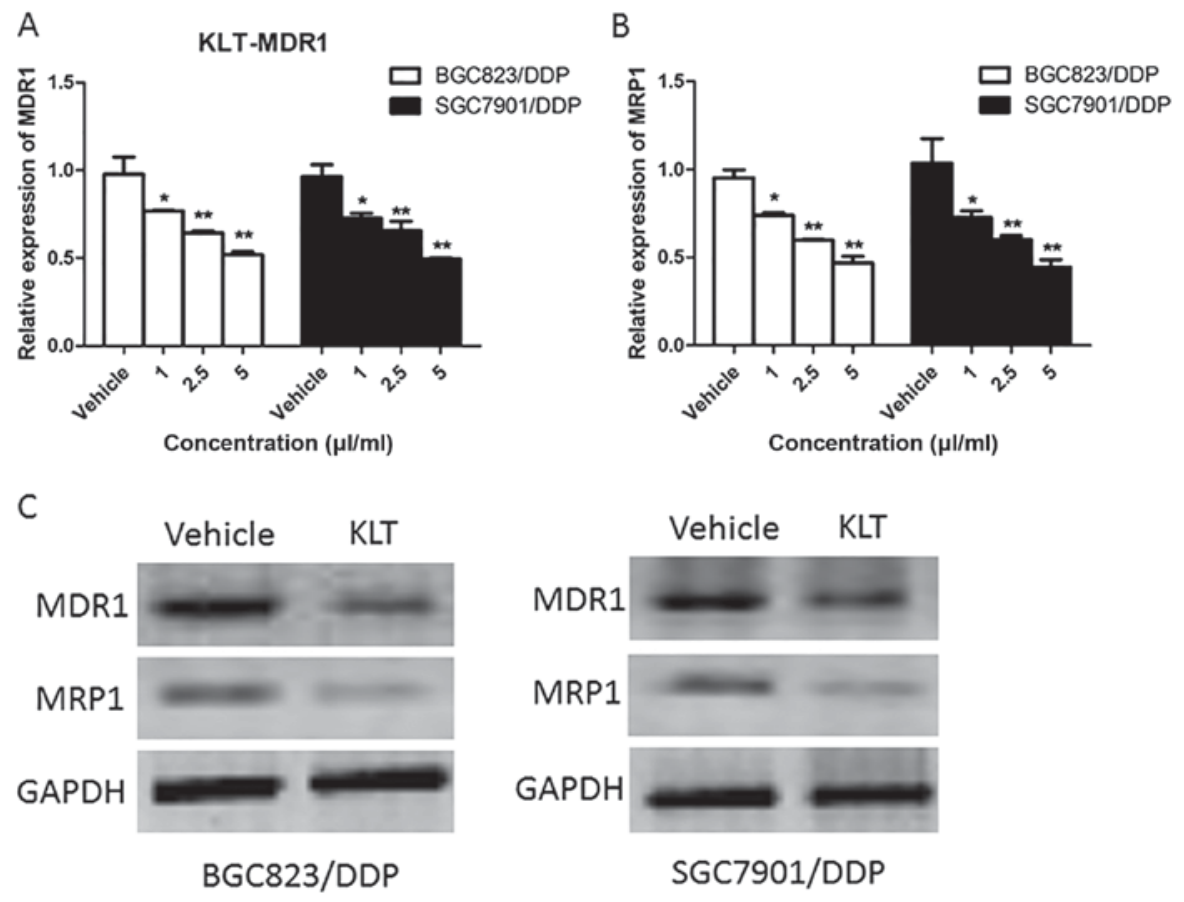

Figure 2. KLT suppressed the expression of drug resistance-related proteins. The mRNA levels of MDR1 (A) and MRP1 (B) were detected by quantitative PCR in BGC823/DPP and SGC7901/DDP cells treated with different concentration (1, 2.5, $5 \mu \mathrm{l} / \mathrm{ml})$ of KLT for $24 \mathrm{~h}$. (C) The protein levels of MDR1 and MRP1 were examined by western blot in BGC823/DPP and SGC7901/DDP cells treated with $2.5 \mu \mathrm{l} / \mathrm{ml}$ of KLT for $48 \mathrm{~h}$. All values are mean \pm SD. ${ }^{*} \mathrm{P}<0.05$ vs. vehicle group; ${ }^{* *} \mathrm{P}<0.01$ vs. vehicle group.

A

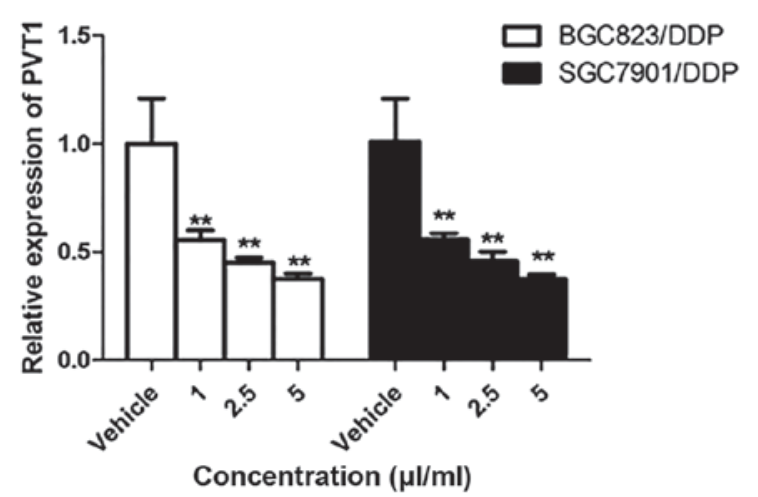

B

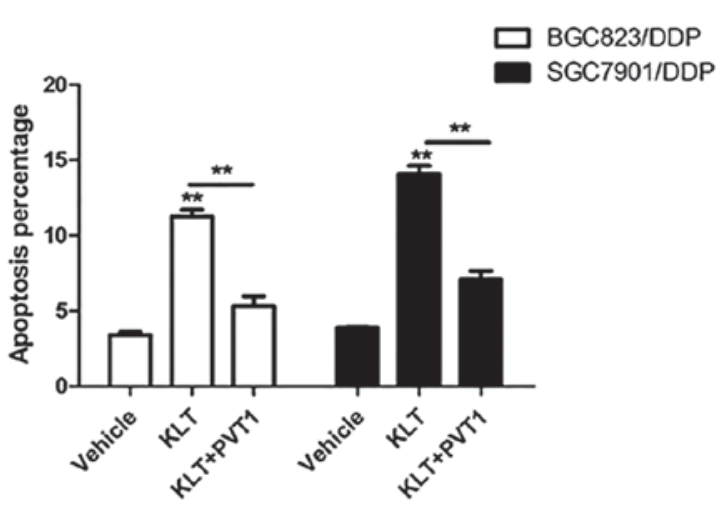

Figure 3. KLT inhibited the expression of PVT1. (A) The level of PVT1 was detected in BGC823/DPP and SGC7901/DDP cells treated with different concentration (1, 2.5 and $5 \mu \mathrm{l} / \mathrm{ml}$ ) of KLT for $24 \mathrm{~h}$. (B) Cell apoptosis was detected in BGC823/DPP and SGC7901/DDP cells pretreated with Ad-PVT1 followed by the treatment of $2.5 \mu \mathrm{l} / \mathrm{ml}$ of KLT for $48 \mathrm{~h}$. All values are mean $\pm \mathrm{SD} .{ }^{* *} \mathrm{P}<0.01 \mathrm{vs}$. vehicle group.

\section{Discussion}

Traditional Chinese medicine has been known to show significant advantages in improving the clinical symptom and the therapeutic efficacy of chemotherapy, as well as in alleviating the side effects of chemotherapy (17). KLT is an antitumor drug widely used clinically in China. It has been found to improve quality of life of cancer patients, strengthen immune function and reduce cancer cachexia. Several researches have aimed to clarify the antitumor mechanism of KLT and demonstrated that KLT primarily blocked the G2/M phase of the cell cycle, inducing cell apoptosis through activating proapoptotic factors, such as p53, Fas and caspase-3 (4).
Nowadays, MDR has been the major obstacle for the treatment of cancers, including the gastric cancer therapy, which mainly leads to the failure of chemotherapies. KLT also ameliorated the MDR of cancers in clinical use. KLT was reported to be effective in reversing MDR of human lung adenocarcinoma cells and increasing the sensitivity of cancer cells to chemotherapeutic agents (18). A network meta-analysis showed that compared with FOLFOX alone, combinations with KLT injection could reduce nausea and vomiting and the incidence of leukopenia (9). Although the efficacy of KLT on MDR has been recognized, the molecular mechanism was not fully understood. In this study, we showed that KLT inhibited the cell viability and promoted the cell 
A

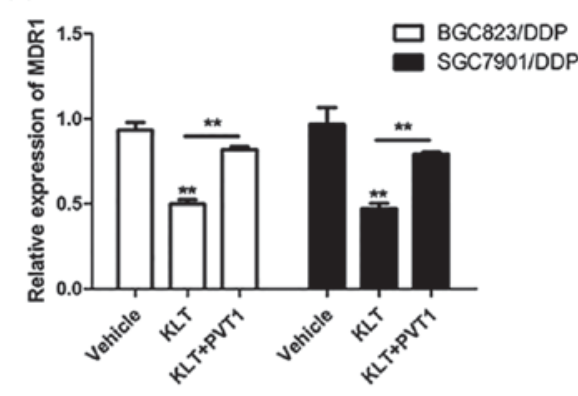

C

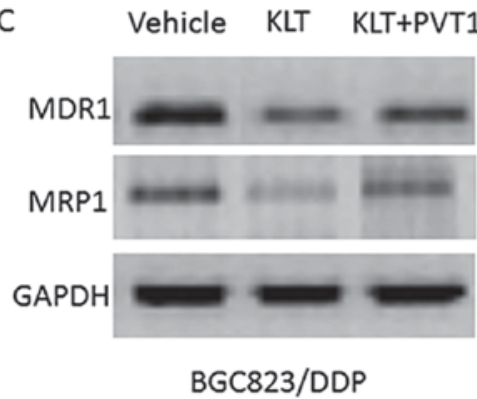

B
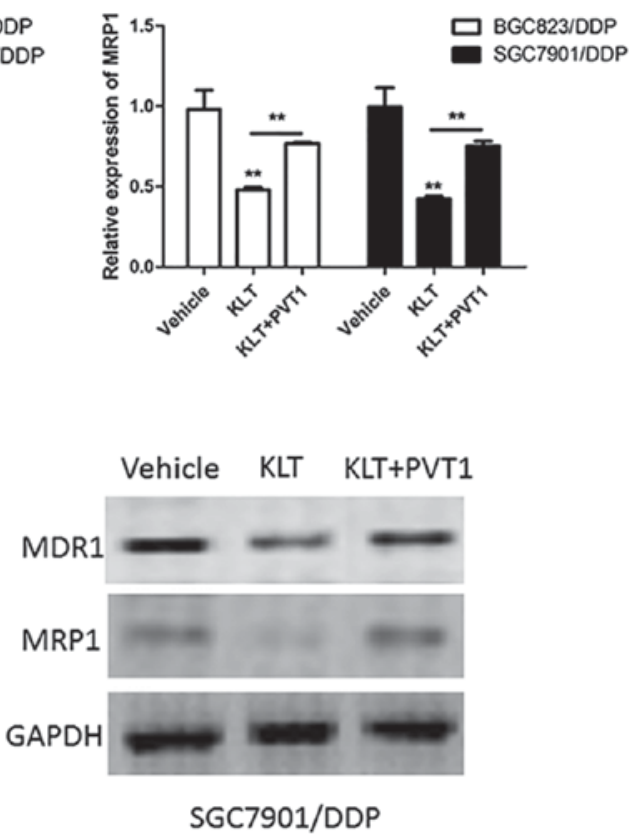

Figure 4. KLT inhibited the expression of drug resistance-related proteins via suppressing the expression of PVT1. BGC823/DPP and SGC7901/DDP cells were transfected Ad-PVT1 and then treated with $2.5 \mu \mathrm{l} / \mathrm{ml}$ of KLT for $24 \mathrm{~h}$. The mRNA level of MDR1 (A) and MRP1 (B) were detected by quantitative PCR (C) The protein level of MDR1 and MRP1 were examined in BGC823/DPP and SGC7901/DDP cells transfected with Ad-PVT1 and treated by $2.5 \mu 1 / \mathrm{ml}$ of KLT for $48 \mathrm{~h}$. All values are mean $\pm \mathrm{SD} .{ }^{* *} \mathrm{P}<0.01 \mathrm{vs}$. vehicle group.

apoptosis of drug-resistant gastric cancer cells. Furthermore, KLT suppressed the expression of MDR1 and MRP1 in BGC823/DPP and SGC7901/DDP cells, which suggested that KLT might attenuate MDR1- and MRP1-regulated MDR in gastric cancer cells.

The MDR1 gene encoded P-glycoprotein (P-gp) which was an efflux pump belonging to the ATP binding cassette transporter superfamily. P-gp efflux pump was able to transport the chemotherapeutic agents out of the cells causing the decreased drug accumulation, then leading to the MDR (19). The mechanism of action of MRP1 for MDR was similar to MDR1 which also belonged to the ATP binding cassette transporter superfamily (20). These data indicated that KLT inhibited anticancer drug resistance by targeting ATP binding cassette transporter family members in gastric cancer.

To further investigate the mechanism through which KLT regulated the expression of MDR1 and MRP1, we explored the effect of KLT on the expression of PVT1, which was observed to promote MDR in gastric cancer cells in our previous study. In several cancers, PVT1 is found to be upregulated in cancer tissues than in adjacent normal tissues, and acts to induce cell proliferation and suppress apoptosis (21-23). In addition, overexpression of PVT1 is involved in cancer resistance to chemotherapy. Similar to the results of our previous study, PVT1 was overexpressed in SGC7901 paclitaxel-resistant cells than in untreated SGC7901 cells (14). In the malignant pleural mesothelioma cell, PVT1 knockdown reduced cell proliferation and increased sensitivity to cisplatin (24). PVT1 was also shown to regulate gemcitabine sensitivity in human pancreatic cancer cells ASPC-1 (25). We showed that KLT inhibited the expression of PVT1 in drug-resistant gastric cancer cells in a time-dependent manner. In addition, PVT1 overexpression reversed the increased percentage of apoptotic cells and the inhibition of the expression of MDR1 and MRP1 induced by KLT. These data indicated that KLT inhibited the expression of MDR1 and MRP1 via suppressing the expression of PVT1.

In conclusion, the results in the present study indicated that KLT inhibited the cell viability and promoted the cell apoptosis of drug-resistant gastric cancer cells. In addition, KLT might attenuate the MDR of gastric cancer cells associating with inhibiting MDR1 and MRP1. Furthermore, KLT inhibited the expression of MDR1 and MRP1 via suppressing the expression of PVT1. The present findings reveal a novel mechanism of KLT function on the MDR of gastric cancer cells, which can potentially be exploited to prevent the MDR of gastric cancer.

\section{Acknowledgements}

This study was supported by the National Natural Science Foundation of China under grant no. 81673736.

\section{References}

1. Xin Y, Yin F, Qi S, Shen L, Xu Y, Luo L, Lan L and Yin Z: Parthenolide reverses doxorubicin resistance in human lung carcinoma A549 cells by attenuating NF- $\kappa$ B activation and HSP70 up-regulation. Toxicol Lett 221: 73-82, 2013.

2. Chen SY, Zheng XW, Cai JX, Zhang WP, You HS, Xing JF and Dong YL: Histone deacetylase inhibitor reverses multidrug resistance by attenuating the nucleophosmin level through PI3K/Akt pathway in breast cancer. Int J Oncol 49: 294-304, 2016.

3. Gillet JP and Gottesman MM: Mechanisms of multidrug resistance in cancer. Methods Mol Biol 596: 47-76, 2010.

4. Lu Y, Li CS and Dong Q: Chinese herb related molecules of cancer-cell-apoptosis: A minireview of progress between Kanglaite injection and related genes. J Exp Clin Cancer Res 27: 31, 2008. 
5. Yu F, Gao J, Zeng Y and Liu CX: Inhibition of Coix seed extract on fatty acid synthase, a novel target for anticancer activity. J Ethnopharmacol 119: 252-258, 2008.

6. Qi F, Li A, Inagaki Y, Gao J, Li J, Kokudo N, Li XK and Tang W: Chinese herbal medicines as adjuvant treatment during chemoor radio-therapy for cancer. Biosci Trends 4: 297-307, 2010.

7. Zhu L, Yang Z, Wang S and Tang Y: Kanglaite for treating advanced non-small-cell lung cancer: A systematic review. Zhongguo Fei Ai Za Zhi 12: 208-215, 2009 (In Chinese).

8. Li DP: General survey and progress in clinical trials abroad over Kanglaite injection. Chin J Integr Med 10: 233-235, 2004.

9. Wang JC, Tian JH, Ge L, Gan YH and Yang KH: Which is the best Chinese herb injection based on the FOLFOX regimen for gastric cancer? A network meta- analysis of randomized controlled trials. Asian Pac J Cancer Prev 15: 4795-4800, 2014.

10. Zhan YP, Huang XE, Cao J, Lu YY, Wu XY, Liu J, Xu X, Xiang J and Ye LH: Clinical safety and efficacy of Kanglaite ${ }^{\circledR}$ (Coix Seed Oil) injection combined with chemotherapy in treating patients with gastric cancer. Asian Pac J Cancer Prev 13: 5319-5321, 2012

11. Beck-Engeser GB, Lum AM, Huppi K, Caplen NJ, Wang BB and Wabl M: Pvt1-encoded microRNAs in oncogenesis. Retrovirology 5: 4, 2008.

12. Guan Y, Kuo WL, Stilwell JL, Takano H, Lapuk AV, Fridlyand J, Mao JH, Yu M, Miller MA, Santos JL, et al: Amplification of PVT1 contributes to the pathophysiology of ovarian and breast cancer. Clin Cancer Res 13: 5745-5755, 2007.

13. Takahashi Y, Sawada G, Kurashige J, Uchi R, Matsumura T, Ueo H, Takano Y, Eguchi H, Sudo T, Sugimachi K, et al: Amplification of PVT-1 is involved in poor prognosis via apoptosis inhibition in colorectal cancers. Br J Cancer 110: 164-171, 2014.

14. Ding J, Li D, Gong M, Wang J, Huang X, Wu T and Wang C: Expression and clinical significance of long non-coding RNA-PVT1 in human gastric cancer. Onco Targets Ther 7: 1625-1630, 2014.

15. Liu E, Liu Z, Zhou Y, Mi R and Wang D: Overexpression of long non-coding RNA PVT1 in ovarian cancer cells promotes cisplatin resistance by regulating apoptotic pathways. Int J Clin Exp Med 8: 20565-20572, 2015.
16. Zhang XW, Bu P, Liu L, Zhang XZ and Li J: Overexpression of long non-coding RNA PVT1 in gastric cancer cells promotes the development of multidrug resistance. Biochem Biophys Res Commun 462: 227-232, 2015.

17. Qi F, Zhao L, Zhou A, Zhang B, Li A, Wang Z and Han J: The advantages of using traditional Chinese medicine as an adjunctive therapy in the whole course of cancer treatment instead of only terminal stage of cancer. Biosci Trends 9: 16-34, 2015

18. Pin-Tian L, Kun Z, Ya-zhen W and Bin L: Effect of Kanglaite Injection associated with Cisplatin in lung adno carcinoma cells A549. Chin Traditional Patent Med 33: 393-396, 2011.

19. Minchinton AI and Tannock IF: Drug penetration in solid tumours. Nat Rev Cancer 6: 583-592, 2006.

20. Cole SP: Targeting multidrug resistance protein 1 (MRP1, ABCC1): Past, present, and future. Annu Rev Pharmacol Toxicol 54: 95-117, 2014.

21. Cui D, Yu CH, Liu M, Xia QQ, Zhang YF and Jiang WL: Long non-coding RNA PVT1 as a novel biomarker for diagnosis and prognosis of non-small cell lung cancer. Tumor Biol 37: 4127-4134, 2016.

22. Ding C, Yang Z, Lv Z, DU C, Xiao H, Peng C, Cheng S, Xie H, Zhou L, Wu J and Zheng S: Long non-coding RNA PVT1 is associated with tumor progression and predicts recurrence in hepatocellular carcinoma patients. Oncol Lett 9: 955-963, 2015.

23. Zhou Q, Chen J, Feng J and Wang J: Long noncoding RNA PVT1 modulates thyroid cancer cell proliferation by recruiting EZH2 and regulating thyroid-stimulating hormone receptor (TSHR). Tumour Biol 37: 3105-3113, 2016.

24. Riquelme E, Suraokar MB, Rodriguez J, Mino B, Lin HY, Rice DC, Tsao A and Wistuba II: Frequent coamplification and cooperation between C-MYC and PVT1 oncogenes promote malignant pleural mesothelioma. J Thorac Oncol 9: 998-1007, 2014.

25. You L, Chang D, Du HZ and Zhao YP: Genome-wide screen identifies PVT1 as a regulator of Gemcitabine sensitivity in human pancreatic cancer cells. Biochem Biophys Res Commun 407: 1-6, 2011. 